\title{
OPEN A novel introgression line collection to unravel the genetics of climacteric ripening and fruit quality in melon
}

Lara Pereira ${ }^{1,3}$, Miguel Santo Domingo ${ }^{1,3}$, Jason Argyris ${ }^{1,2}$, Carlos Mayobre $^{1}$, Laura Valverde ${ }^{1}$, Ana Montserrat Martín-Hernández ${ }^{1,2}$, Marta Pujol ${ }^{1,2}$ \& Jordi Garcia-Mas ${ }^{1,2}$

Introgression lines are valuable germplasm for scientists and breeders, since they ease genetic studies such as QTL interactions and positional cloning as well as the introduction of favorable alleles into elite varieties. We developed a novel introgression line collection in melon using two commercial European varieties with different ripening behavior, the climacteric cantalupensis 'Védrantais' as recurrent parent and the non-climacteric inodorus 'Piel de Sapo' as donor parent. The collection contains 34 introgression lines, covering $99 \%$ of the donor genome. The mean introgression size is $18.16 \mathrm{Mb}$ and $\sim 3$ lines were obtained per chromosome, on average. The high segregation of these lines for multiple fruit quality traits allowed us to identify 27 OTLs that modified sugar content, altered fruit morphology or were involved in climacteric ripening. In addition, we confirmed the genomic location of five major genes previously described, which control mainly fruit appearance, such as mottled rind and external color. Most of the QTLs had been reported before in other populations sharing parental lines, while three QTLs (EAROQP11.3, ECDQP11.2 and FIRQP4.1) were newly detected in our work. These introgression lines would be useful to perform additional genetic studies, as fine mapping and gene pyramiding, especially for important complex traits such as fruit weight and climacteric ripening.

Understanding the genetics beyond interesting traits, from human diseases to crop yield, has been one of the main goals of modern science. Genetic variation is studied and correlated with phenotypes, in order to identify the genomic regions controlling traits of interest. In plants, due to the ease of crossing, many types of segregating populations can be developed depending on the goal of the research and the availability of time and resources. In species with short generation periods, as cereals and vegetable crops, populations with increased complexity are usually developed, as Recombinant Inbred Line (RIL) ${ }^{1}$, Nested Association Mapping ${ }^{2}$, Multi-parent Advanced Generation Intercrosses ${ }^{3}$ and Introgression Line (IL $)^{4,5}$ populations. IL collections are immortal lines that share a high proportion of genetic background from a recurrent parent, differing only in a specific region of the genome (introgression), inherited from the donor parent. Ideally, the complete genome of the donor parent is covered by the ILs conforming the collection. They are considered a powerful resource since, besides Quantitative Trait Loci (QTL) mapping experiments, they allow to perform subsequent specific studies as QTL validation ${ }^{6}$, QTL interactions ${ }^{7,8}$ and fine mapping ${ }^{9,10}$.

IL populations (also referred as Near Isogenic Lines, NILs) have been used as genetic resources in multiple species for several decades ${ }^{11-13}$, from model plants as Arabidopsis thaliana ${ }^{14}$, rice $^{15}$ and tomato ${ }^{16}$ to less studied and even orphan crops, as strawberry ${ }^{17}$, peach ${ }^{18}$ and groundnut ${ }^{19}$. They have proven their efficiency to map and characterize traits related with disease resistance, plant architecture and fruit morphology and quality. Furthermore, they have been commonly used in breeding programs to introduce desired exotic alleles from donor accessions, where the recurrent parent is typically an elite line.

Generally, ILs are developed by genotyping the target introgression as well as the recurrent parent background with molecular markers. The rapid increase in marker availability and high-throughput genotyping techniques have changed the methods used to develop these populations. Recently, the trend has been to minimize the number of needed backcrosses by increasing the size of the initial screened populations and the number of

${ }^{1}$ Centre for Research in Agricultural Genomics (CRAG) CSIC-IRTA-UAB-UB, Edifici CRAG, Campus UAB, 08193 Bellaterra, Barcelona, Spain. ${ }^{2}$ Institut de Recerca i Tecnologia Agroalimentàries (IRTA), Edifici CRAG, Campus UAB, 08193 Bellaterra, Barcelona, Spain. ${ }^{3}$ These authors contributed equally: Lara Pereira and Miguel Santo Domingo. ${ }^{\bowtie}$ email: marta.pujol@irta.cat; jordi.garcia@irta.cat 
markers, in order to decrease the number of non-desired contaminations in narrow non-genotyped regions of the genome $\mathrm{e}^{5,17,18}$.

IL populations have been developed and widely used throughout the last years in melon ${ }^{4,9,10,20-26}$. Melon is a diploid species with a relatively small genome $(450 \mathrm{Mb})$ and $2 \mathrm{n}=2 \mathrm{x}=24^{27}$. Melon has become an alternative model species to study certain traits, as climacteric ripening ${ }^{28}$ and sex determination ${ }^{29}$. An IL collection, developed from a cultivated variety (spp. melo) as recurrent parent and an exotic Korean accession (spp. agrestis) as donor parent ${ }^{4}$, was used to fine map and clone two genes controlling climacteric ripening ${ }^{9}$ and resistance to Cucumber Mosaic Virus ${ }^{10}$. In addition, pyramiding of multiple QTLs controlling or modulating the same trait was achieved through crosses of several ILs; e.g., the QTL ETHQV6.3, corresponding to CmNAC-NOR ${ }^{9}$ and involved in climacteric ripening, interacts with a second QTL (ETHQB3.5), leading to a more intense climacteric phenotype when both introgressions are combined in the same $\mathrm{IL}^{23}$.

Most of the populations developed in melon have been funded by a cross between an elite cultivar and an exotic accession. A recent study using a RIL population between two European cultivars, "Védrantais" (Ved), a typical French variety belonging to the cantalupensis group, and "Piel de Sapo" (PS), a Spanish variety from the inodorus group, showed segregation for traits related with fruit quality and climacteric ripening ${ }^{30-32}$. Climacteric ripening is a complex and polygenic trait that partially determines the overall fruit quality. Melon is one of the few species where both climacteric and non-climacteric varieties coexist, offering an excellent model to dissect the genetics of the trait ${ }^{28}$. Climacteric ripening is defined by an autocatalytic synthesis of the plant hormone ethylene at the onset of the ripening stage, followed by a peak of respiration ${ }^{33}$. In climacteric melons, these biochemical signals trigger multiple phenotypic changes such as chlorophyll degradation, production of several volatiles that lead to a sweet aroma, abscission layer formation in the pedicel and degradation of cell wall resulting in a loss of flesh firmness ${ }^{34}$.

The aim of this work was to develop an IL collection using Ved as recurrent parent and PS as donor parent in order to identify and validate QTLs controlling fruit quality and climacteric ripening in melon. The novelty of this IL collection is that both the recurrent (Ved) and the donor (PS) are elite cultivars that segregate for many traits. Ved is a medium-size rounded melon, with rind sutures, orange flesh, highly aromatic and climacteric ripening, while PS has bigger size, with elongated shape, mottled rind, white flesh, and non-climacteric ripening. This advanced germplasm resource would be useful in the future to further characterize these genetic factors through fine mapping and QTL interaction studies.

\section{Results and discussion}

Parent phenotypes. Two commercial lines, Ved and PS, were selected as parents to develop the IL collection due to the segregation of several relevant traits among them, such as ripening behavior and external appearance. They represent the two most common and consumed melons in Europe. Ved produces a round, medium-size fruit, whereas PS fruits are elongated and its fruit weight is higher (Supplementary Fig. S1A). The soluble solid content and fruit firmness are slightly higher in PS although not significantly different from Ved. The external appearance of the fruit also varies: Ved presents white/cream rind with marked sutures and PS mottled green rind (Supplementary Fig. S1B). The flesh color is orange in Ved and white in PS.

Interestingly, these two varieties represent the two extremes of ripening behavior in melon ${ }^{31}$. Ved is a typical climacteric variety, presenting the characteristic ethylene peak at the onset of ripening closely followed by classical phenotypic consequences such as intense sweet aroma, abscission layer formation, chlorophyll degradation and loss of flesh firmness. PS is completely non-climacteric, the ethylene production during ripening remains undetectable and it lacks the phenotypic changes associated to climacteric ripening, except loss of flesh firmness, a trait partially ethylene-independent ${ }^{34}$.

Development of the PS IL collection. IL collections are a highly valuable resource for both research and breeding, although their development is more complex and expensive than other populations. In the last years, many IL collections have been described for different species, including melon, which have been successfully used to map and characterize QTLs ${ }^{35-37}$. Here, we developed a new IL collection to gain insight about the genetic control of fruit quality.

In the $\mathrm{BC}_{1}$ population, the number of introgressions ranged from 4 to 17 per line, with a mean of 11.5 and a median of 12, indicating that on average one recombination per chromosome and meiosis had occurred. The use of marker-assisted selection in the first BC generation reduced substantially the risk of carrying nondetected contaminations. The percentage of Ved genome ranged from 13.0 to $80.1 \%$, with an average of $48.7 \%$. The observed heterozygosity was $\mathrm{H}_{0}=0.46$ and 16 SNPs showed significant segregation distortion. The highest segregation distortion was observed in the centromeric region of chromosome $12\left(\chi^{2}=43.3\right)$, which presented a minimum observed heterozygosity of 0.31 . A set of $25 \mathrm{BC}_{1}$ plants $(7.8 \%)$ were selected, presenting a mean of 8 introgressions and $62.2 \%$ of Ved background genome. After two more cycles of backcross and marker assisted selection to $\mathrm{BC}_{2}$ and $\mathrm{BC}_{3}$ (Fig. 1), the number of introgressions in the 64 selected lines was notably reduced to a mean of 2.6 introgressions and they carried an $85.7 \%$ of Ved genome on average. In the next generation, a set of 100 plants were selected, carrying 1.8 introgressions and $88.3 \%$ of Ved genome on average, and most of the final ILs were recovered from their progenies. Due to various complications (misbehaved SNPs, loss of plants due to pests or bad fruit set), a $6 \%$ of PS genome remained uncovered in the IL collection. Lines from previous generations were used to obtain additional ILs, and three and two of them were phenotyped in 2019 and 2020, respectively.

The most remarkable parameters for an IL collection are the number of ILs per chromosome, which is directly correlated with the resolution and with the required resources for phenotyping, the size of the introgressions, which also defines the resolution, and the number of generations needed to obtain the ILs, which is related with 


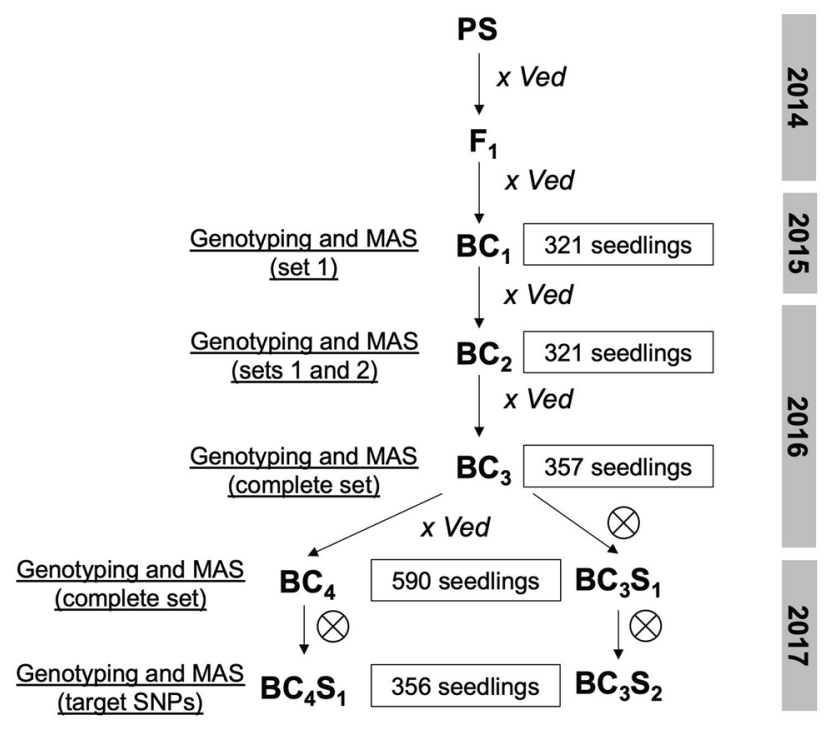

Figure 1. Breeding scheme followed to develop the IL collection.

undesired introgressions in non-target regions. The PS IL collection, comprised by 34 lines, covered the complete genome of PS except the last $3.8 \mathrm{Mb}$ of chromosome 3 (Fig. 2A and Supplementary Table S1). A mean of 2.8 ILs per chromosome was obtained, with a large range of introgression length from 2.46 to $35.32 \mathrm{Mb}$ (Supplementary Table S2). Considering the overlapping of introgressions from different ILs, we could define 42 genomic bins. In comparison to other IL collections, this is an intermediate size, below the 6 ILs per chromosome obtained in a strawberry IL collection ${ }^{17}$ but higher than the 1.3 ILs per chromosome from other recently described melon IL collections ${ }^{20,21}$ (Supplementary Table S3). The size in genetic distance units, inferred from a genetic map in a RIL population using the same parents ${ }^{30}$, ranged from 14.7 to $129.2 \mathrm{cM}$, with an average of $62.8 \mathrm{cM}$. As we could expect, the introgressions in the telomeric regions were smaller in terms of physical distance but larger in genetic distance, as opposed to the introgressions covering the pericentromeric regions (Fig. 2A). The size of the introgressions was slightly higher than the genetic distance value obtained in other IL collections (mean of $38.7 \mathrm{cM}$ ), but similar in physical distance $e^{4,5,17,20}$. This discordance could be attributed to the strategy used to select the SNPs for the marker-assisted selection, which was mainly based on physical distances (markers homogeneously distributed across the chromosomes). The number of generations used to obtain the ILs in our work was generally six $\left(\mathrm{BC}_{3} \mathrm{~S}_{2}\right.$ and $\left.\mathrm{BC}_{4} \mathrm{~S}_{1}\right)$, as in most other IL collections (Supplementary Table S3). The percentage of covered PS genome is $\sim 99 \%$, slightly higher than in other IL collections.

QTL mapping. Fruit quality. Several traits contributing to fruit quality were segregating in the IL collection, including several external characteristics of the fruit as color and morphology (Fig. 2B, Supplementary Table S4). Qualitative phenotypes determining the external appearance of the fruit were already appreciated during the development of the IL collection. The mapping of these traits to the corresponding genomic region was easily performed even in pre-ILs due to its simple inheritance and high heritability, and the phenotyping of the complete collection performed in 2018, 2019 and 2020 confirmed the preliminary results.

PS2.2 presented mottled rind, observed as dark green spots during early stages of fruit development and turning to bright yellow spots in ripe fruits (Fig. 3A). This trait is mainly controlled by the major gene $M t-2$, delimited to the interval $24.77-27.06 \mathrm{Mb}$ on chromosome 2. $\mathrm{Mt}$-2 was also mapped to chromosome 2 in previous studies ${ }^{30,38}$.

PS7.1 and PS7.2 showed a dark green external color during fruit development as PS, contrasting to the rest of the IL collection which shared the white external color present in Ved. Similarly, this trait is mainly determined by one unique gene, previously described and designated $W i^{39}$, delimited by the overlapping region of both ILs, which is located within 2.62-20.72 bp. This interval colocalized with the region identified in the Ved $\times$ PS RIL population $^{30}$.

PS10.1 fruits were white during fruit development as Ved but turned to a light-yellow during fruit ripening instead of cream (Fig. 3B). The interval of the gene primarily determining this trait $(0-5.10 \mathrm{Mb}$ on chromosome 10), contains $C m K F B$, which controls flavonoids biosynthesis in melon rind ${ }^{40}$.

PS11.1 and PS11.3 presented smooth rind, without sutures (Fig. 3C), in contrast to Ved and the rest of the IL collection. The responsible gene could be mapped to the interval shared by both lines on chromosome 11, from 21.26 to $29.79 \mathrm{Mb}$. MELO3C019694, located within the introgressions of PS11.1 and PS11.3, was proposed as responsible for the presence of sutures, due to a delay in gene expression during the initial stages of fruit development ${ }^{41}$. The same trait has been studied in other cucurbits, especially in Cucurbita pepo ${ }^{42}$, but the biological process causing the sutures has not been described yet.

PS9.4 was the only IL in our collection without orange flesh (Fig. 3D); however, unlike the donor parent PS, PS9.4 flesh is green. This phenotype is explained by the oligogenic inheritance of flesh color in ripe fruits. Three 
A
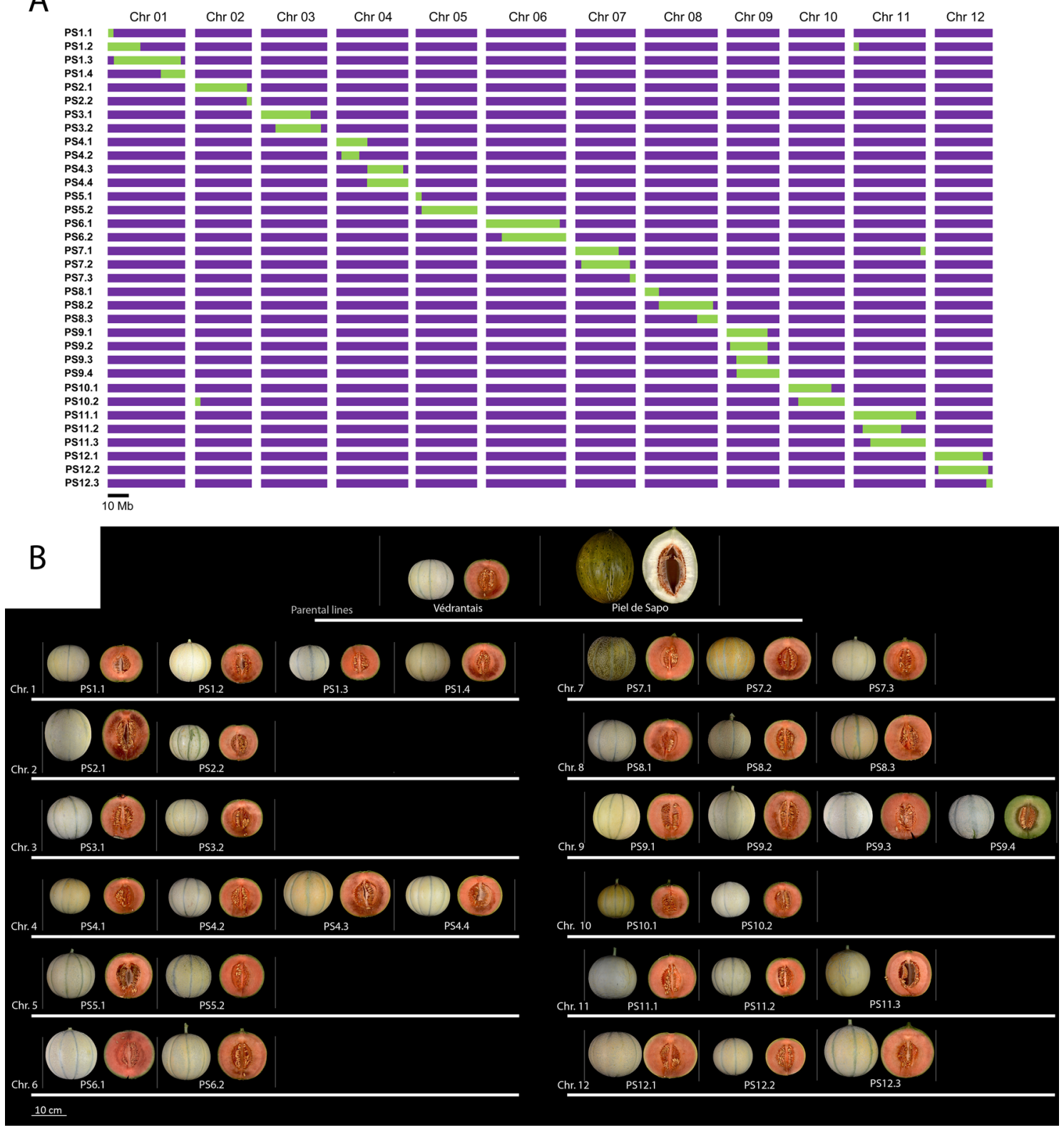

Figure 2. The IL collection. (A) Genotypic characterization of the IL collection. Purple represents the genotype of the recurrent parent Ved and green, the genotype of the donor parent PS. (B) Representative images of the ILs.

phenotypes, orange, white and green flesh, can be observed depending on the allelic combination of two genes that act epistatically. A dominant gene on chromosome 9 already cloned, $\mathrm{Gf}$ or $\mathrm{CmOr}^{43}$, controls the presence of orange color, conferred by a high content of carotenoids and is located in the position $21.68 \mathrm{Mb}$ of chromosome 9 (version v3.6.1 of the melon genome). The non-orange allele, which is recessive, allows the manifestation of the second gene, called $W f$, to control white (dominant) or green (recessive) flesh color ${ }^{44-46}$. Wf has been mapped using several populations to an interval of $5 \mathrm{Mb}$ on chromosome $8^{45,46}$ and two genes, MELO3C003069 and $M E L O 3 C 003097$ have been proposed as possible candidate genes ${ }^{41,44}$. Ved genotype is Gf/Gf $w f / w f$ and PS genotype is $g f / g f W f / W f$; therefore, the genotype of IL PS9.4, carrying a PS introgression in Gflocus, is the double recessive $g f / g f w f / w f$, leading to green color instead of the white color observed in PS. In addition to the visual inspection of flesh color, we also quantified CIELAB color parameters in Tomato Analyzer $4.0^{47}$. We then used the FC index (see "Materials and methods"), representing color differences between the ILs and Ved, with the goal of identifying additional minor QTLs. However, only the ILs carrying the two genes mentioned above were significantly different from Ved (Fig. 4A).

PS8.2 was the only IL in which soluble solid content significantly differed from Ved, with $10.59^{\circ}$ Brix on average, representing an increase of $\sim 34 \%$ compared to Ved (Fig. 4B, Table 1). This QTL was already described in the Ved $\times$ PS RIL population ${ }^{30}$. PS5.1, carrying an introgression from the beginning to $2.83 \mathrm{Mb}$ of chromosome 


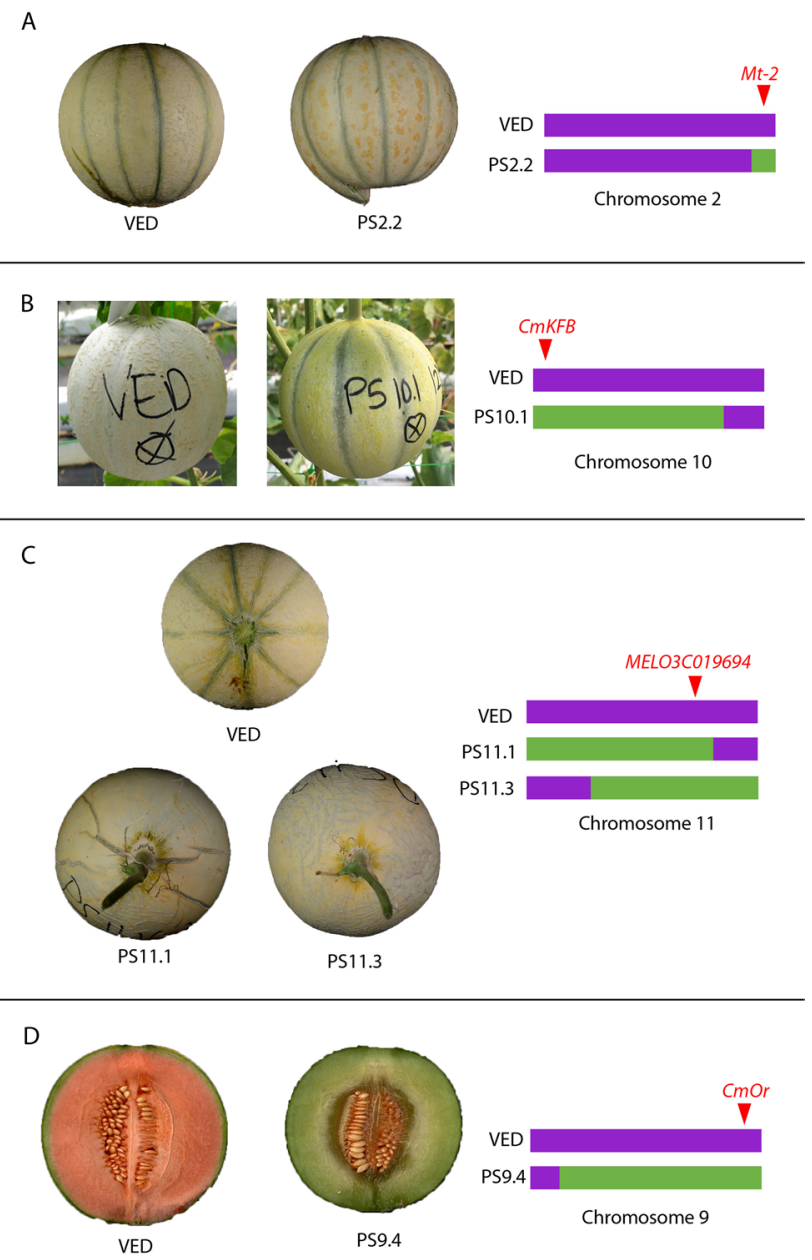

Figure 3. Segregation of qualitative traits and genomic location of the responsible major gene (A) Mottled rind (B) Yellowing of the rind (C) Presence of sutures (D) Flesh color of mature fruits.
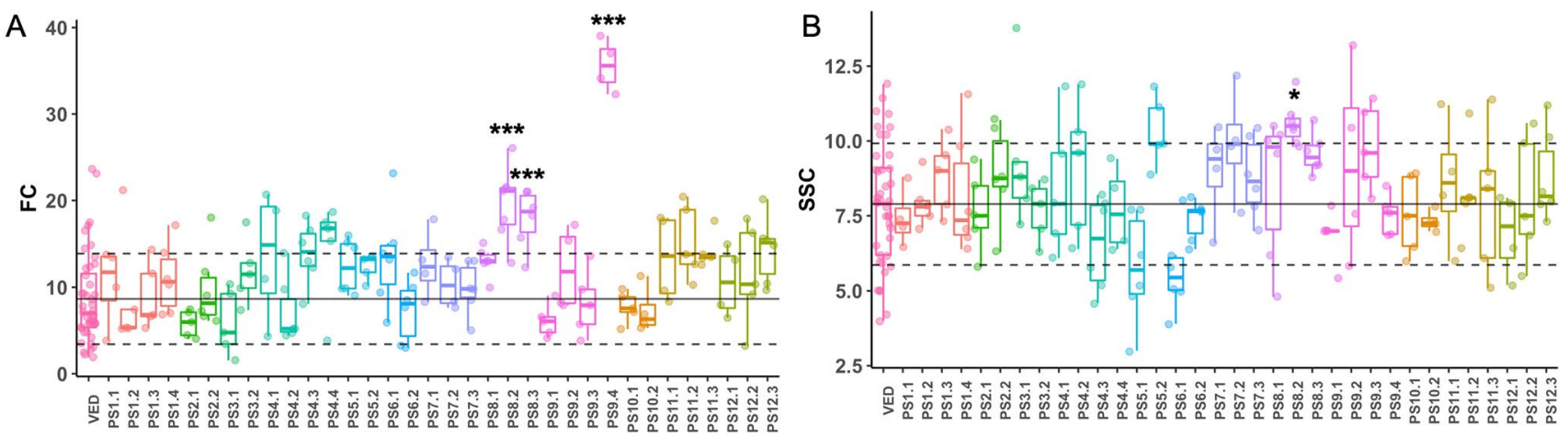

Figure 4. Boxplot representing FC and SSC values for ILs. Each dot represents a replicate, the black solid line corresponds to the average and the grey dashed lines to the average \pm SD of the recurrent parent Ved. Significant differences are marked with asterisks: ${ }^{*}<0.05,{ }^{* *}<0.01,{ }^{* *}<0.001$.

5, reduced soluble solid content $34.3 \%$ when compared to Ved, dropping from $8.78^{\circ}$ to $5.77^{\circ}$ Brix, although it was not significant. This region in chromosome 5 colocalizes with a previously described QTL on chromosome 5 , sscqsc5. $1^{37}$; however, the PS allele, conferring lower soluble solid content in our IL collection, had the opposite effect in PS $\times$ "Trigonus" and PS $\times$ "Songwhan Charmi" 37 .

Fruit morphology. Fruit weight is one of the most relevant traits for the industry since it defines yield and also drives consumer's preferences. Although a few genes controlling fruit weight have been cloned in the model 


\begin{tabular}{|c|c|c|c|c|c|c|c|c|}
\hline Trait & QTL & IL & Chr & $\begin{array}{c}\text { Genomic interval } \\
(\mathrm{Mb})\end{array}$ & $\begin{array}{c}\mathrm{IL} \\
\text { mean }\end{array}$ & $\begin{array}{l}\text { Ved } \\
\text { Mean }\end{array}$ & $\begin{array}{c}\% \\
\text { difference }^{\mathrm{a}}\end{array}$ & $\begin{array}{r}\text { p-value } \\
\text { (Dunett } \\
\text { test) }\end{array}$ \\
\hline SSC & SSCQP8.2 & PS8.2 & 8 & $6.89-25.00$ & 10.59 & 7.9 & 34.05 & 0.0153 \\
\hline \multirow{2}{*}{ FW } & $F W Q P 2.2$ & PS2.2 & 2 & $24.77-27.06$ & 435 & \multirow{2}{*}{717.04} & -39.33 & 0.0184 \\
\hline & $F W Q P 4.1$ & PS4.1 & 4 & $0.00-2.44 / 10.97-14.92$ & 394.8 & & -44.94 & 0.0081 \\
\hline \multirow{2}{*}{ FA } & FAQP4.1 & PS4.1 & 4 & $0.00-2.44 / 10.97-14.92$ & 62.66 & 99.05 & -36.74 & 0.0218 \\
\hline & FAQP10.1 & $\mathrm{PS} 10.1$ & 10 & $0.00-5.25$ & 69.97 & 104.76 & -33.2 & 0.0034 \\
\hline \multirow{2}{*}{ FP } & $F P Q P 4.1$ & PS4.1 & 4 & $0.00-2.44 / 10.97-14.92$ & 29.99 & 39.11 & -23.32 & 0.003 \\
\hline & $F P Q P 10.1$ & PS10.1 & 10 & $0.00-5.25$ & 32.15 & 39.93 & -19.48 & 0.0011 \\
\hline \multirow{2}{*}{ FL } & FLQP4.1 & PS4.1 & 4 & $0.00-2.44 / 10.97-14.92$ & 8.38 & 10.87 & -22.91 & 0.0144 \\
\hline & FLQP10.1 & PS10.1 & 10 & $0.00-5.25$ & 9.07 & 11.37 & -20.23 & 0.0091 \\
\hline \multirow[t]{2}{*}{ FWI } & FWIQP4.1 & PS4.1 & 4 & $0.00-2.44 / 10.97-14.92$ & 9.36 & 11.43 & -18.11 & 0.0088 \\
\hline & FWIQP10.1 & PS10.1 & 10 & $0.00-5.25$ & 9.54 & 11.37 & -16.1 & $<0.0001$ \\
\hline \multirow{2}{*}{ FS } & $F S Q P 2.1$ & PS2.1 & 2 & $0.00-16.42$ & 1.1 & 0.95 & 15.79 & $<0.0001$ \\
\hline & FSQP4.4 & PS4.4 & 4 & $27.25-34.32$ & 0.87 & 0.98 & -11.22 & 0.03 \\
\hline \multirow{2}{*}{ EALF } & EALFQP6.2 & PS6.2 & 6 & $7.47-38.30$ & 37.6 & \multirow{2}{*}{33.64} & 11.77 & 0.0052 \\
\hline & EALFQP8.2 & PS8.2 & 8 & $6.89-25.00$ & 41.75 & & 24.11 & $<0.0001$ \\
\hline \multirow{3}{*}{ EARO } & EAROQP6.2 & PS6.2 & 6 & $7.47-38.30$ & 36.67 & \multirow{3}{*}{33.55} & 9.3 & 0.0062 \\
\hline & EAROQP8.2 & PS8.2 & 8 & $6.89-25.00$ & 38.67 & & 15.26 & $<0.0001$ \\
\hline & EAROQP11.3 & PS11.3 & 11 & $8.00-34.46$ & 29.8 & & -11.18 & 0.0012 \\
\hline \multirow[t]{2}{*}{ ECD } & $E C D Q P 11.2$ & PS11.2 & 11 & $8.00-21.26$ & 29.5 & \multirow[t]{2}{*}{33.73} & -12.54 & 0.0321 \\
\hline & & PS11.3 & 11 & & 29.8 & & -11.65 & 0.0244 \\
\hline \multirow[t]{3}{*}{ HAR } & HARQP 2.2 & PS2.2 & 2 & $24.77-27.06$ & 40.33 & \multirow[t]{3}{*}{36.29} & 11.13 & 0.0254 \\
\hline & HARQP6.2 & PS6.2 & 6 & $7.47-38.30$ & 42.33 & & 16.64 & 0.0385 \\
\hline & HARQP8.2 & PS8.2 & 8 & $6.89-25.00$ & 45.5 & & 25.38 & $<0.0001$ \\
\hline \multirow{6}{*}{ FIR } & $F I R Q P 2.1$ & PS2.1 & 2 & $0.00-16.42$ & 0.68 & \multirow{6}{*}{2.61} & -73.95 & $<0.0001$ \\
\hline & FIRQP4.1 & PS4.1 & 4 & $0.00-2.45 / 10.97-14.92$ & 4.26 & & 63.22 & 0.0109 \\
\hline & FIRQP8.2 & PS8.2 & 8 & $6.89-25.00$ & 4.44 & & 70.12 & 0.0181 \\
\hline & FIRQP8.3 & PS8.3 & 8 & $25.00-34.77$ & 4.3 & & 64.75 & 0.0835 \\
\hline & FIRQP12.2 & $\mathrm{PS} 12.2$ & 12 & $22.98-25.89$ & 4.18 & & 60.15 & 0.0192 \\
\hline & & PS12.3 & 12 & & 4.04 & & 54.79 & 0.0221 \\
\hline
\end{tabular}

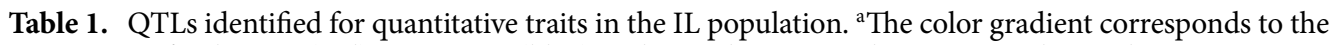
percentage of reduction (red) or increase (blue) in the IL phenotype when compared to Ved.

fruit crop tomato ${ }^{48-50}$, the genetic basis of the trait in melon remains poorly understood. Several QTLs have been described in different populations through both QTL mapping and genome-wide association studies, however the underlying genes responsible of the phenotype remain unknown ${ }^{51}$. In the PS IL population, we identified two QTLs affecting fruit weight, carried by ILs PS2.2 and PS4.1 (Fig. 5). PS4.1 was also significantly different to Ved in other size-related traits as fruit area, fruit perimeter, fruit length and fruit width. In addition, PS10.1, evaluated in 2019, presented lower size overall and was significantly different from Ved for all size-related traits except fruit weight (Table 1 and Supplementary Fig. S2).

PS2.2 borne fruits with an average weight of $435 \mathrm{~g}, 39.3 \%$ reduction as compared to Ved (Table 1, Fig. 5 and Supplementary Fig. S3). FWQP2.2 was delimited to the distal region of chromosome 2, from 24.77 to $27.06 \mathrm{Mb}$. No QTLs were identified for the other size-related traits in the same genomic region, although some of the fruits actually presented lower values (Fig. 5); these traits were more variable than fruit weight, possibly causing a lack of statistical power. A meta-QTL for fruit weight was defined previously on chromosome 2, colocalizing with the gene $a$, which causes sex determination in female flower. Thus, in those populations the fruit weight difference may be a consequence of sex determination ${ }^{51,52}$. Although the QTL described in our IL collection does not 

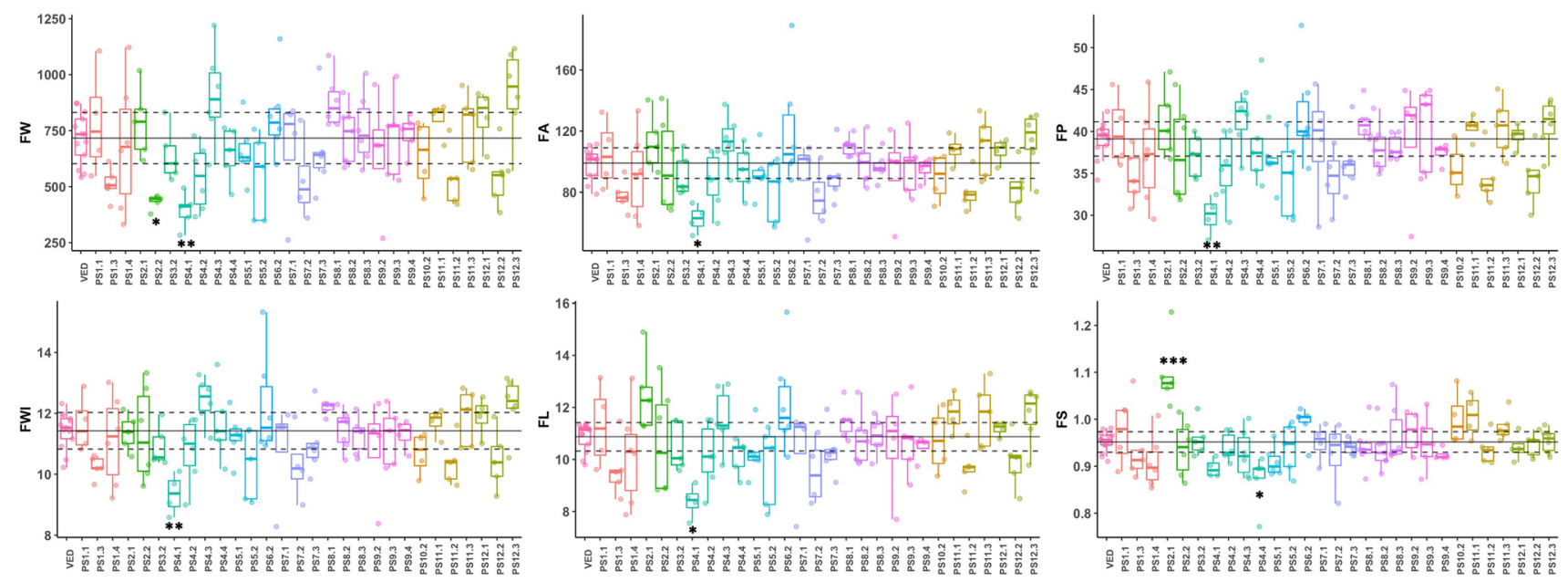

Figure 5. Boxplot representing the values of morphological traits for ILs in 2018. Each dot represents a replicate, the black solid line corresponds to the average and the grey dashed lines to the average \pm SD of the recurrent parent Ved. Significant differences are marked with asterisks: ${ }^{*}<0.05,{ }^{* *}<0.01,{ }^{* * *}<0.001$.

colocalize with a (MELO3C015444), indicating that the underlying gene has to be distinct, another QTL has been also reported at the distal part of chromosme 2 in a RNA-Seq-based QTL mapping ${ }^{44}$.

PS4.1 fruits weighed $394.8 \mathrm{~g}$ and showed a significantly smaller size than Ved (Table 1 and Fig. 5). The reduction in average values for the IL as compared to Ved was about $44.9 \%$ for fruit weight, $36.7 \%$ for fruit area, $23.3 \%$ for fruit perimeter, $18.1 \%$ for fruit width and $22.9 \%$ for fruit length. Since both dimensions were similarly decreased, the fruit shape was not affected. PS4.1 covers from the beginning of the chromosome 4 to $14.92 \mathrm{Mb}$; however, PS4.2, which did not show any fruit size difference compared to Ved, spans from 2.44 to $10.97 \mathrm{Mb}$. Therefore, FWQP4.1 could be located either from 0 to $2.44 \mathrm{Mb}$ or from 10.97 to $14.92 \mathrm{Mb}$ (Supplementary Fig. S3). Although we do not know in which part of the introgression is the QTL, another QTL for fruit weight was previously reported in an IL population using PS as recurrent parent ${ }^{24}$, colocalizing with the second part of FWQP4.1, suggesting our QTL might be located in the $10.97-14.97 \mathrm{Mb}$ interval.

PS10.1 carried fruits with a smaller size and a flattened shape in comparison to Ved. Fruit area, fruit perimeter, fruit length and fruit width were reduced between $\sim 16$ and $33 \%$. Fruit weight was also lower than the rest of ILs but was not significant (Supplementary Fig. S2). These QTLs (FAQP10.1, FPQP10.1, FLQP10.1 and FWIQP10.1) were located on chromosome 10 from 0 to $5.25 \mathrm{Mb}$ (Table 1, Supplementary Fig. S3). It is not the first time that a QTL affecting fruit morphology is located on chromosome 10. In a similar study with ILs, but using different recurrent and donor parents, a QTL at the beginning of chromosome 10 was reported affecting fruit weight ${ }^{24}$.

Surprisingly, in these three ILs the alleles from PS were reducing fruit weight. Nevertheless, undoubtedly some alleles contributing to develop large fruits must be present in the PS genome, since its fruit weight is nearly double than Ved fruits. We observed a trend in four ILs which showed a median fruit weight higher than average plus one standard deviation of Ved fruit weight. PS4.3, PS8.1, PS12.1 and PS12.3 fruits weighed 905, 880, 812.82 and $916.67 \mathrm{~g}$, respectively, representing increases in fruit weight between 16.3 and $31.1 \%$ as compared to Ved (Fig. 5). However, the values presented a substantial variation within each line, probably preventing from detecting significant differences. The larger size of these fruits was visually obvious (Fig. 2B) and also other sizerelated traits as fruit area and fruit perimeter showed this trend (Fig. 5).

Two QTLs were identified for fruit shape. PS2.1 presented more elongated fruits, with an average fruit shape index of 1.10 which represents an increase of $15.79 \%$ as compared to Ved. FSQP2.1 was delimited from the beginning of chromosome 2 to position $16.42 \mathrm{Mb}$ (Supplementary Fig. S3). This QTL does colocalize with the locus a previously mentioned ${ }^{52}$. However, both Ved and PS are andromonoecious, indicating that the genetic variation causing fruit shape differences may be due to a different underlying gene. PS4.4 carried flatter fruits with a fruit shape index of 0.87 , a reduction of $11.22 \%$ as compared to Ved (Table 1 and Fig. 5). FSQP4.4 was located on the distal part of chromosome 4, from 27.25 to $34.32 \mathrm{Mb}$ (Supplementary Fig. S3), colocalizing with a recently reported QTL for fruit shape in an $\mathrm{F}_{2}$ melon population ${ }^{53}$.

Curiously, none of the ILs fruit weight QTLs were identified in the Ved $\times$ PS RIL population ${ }^{30}$. The discrepancy may be due to QTL $\times$ QTL interactions, which could make some QTLs indetectable in certain genetic backgrounds. Similarly, fruit weight QTLs detected in the RIL population where not detected in the ILs, but FWQU5.1 and FWQU8.1, would correspond to ILs PS5.2 and PS8.1; PS5.2 presented a high variation between replicates, possibly preventing from detecting a QTL at significant levels. PS8.1, already mentioned, showed an increase in fruit weight of $\sim 180 \mathrm{~g}$, considerably higher to the $92.4 \mathrm{~g}$ additive effect of PS observed in the Ved $\times$ PS RIL population ${ }^{30}$. The high variation between replicates and its limited number could cause lack of statistical power to detect a QTL confidently, but the observed trend in fruit weight suggests that the PS allele in PS8.1 contributes to larger fruits, in agreement with the Ved $\times$ PS RIL population. In addition, a QTL for fruit weight was also detected in the same region of chromosome 8 in an $\mathrm{F}_{2}$ obtained by crossing a wild and a cultivated agrestis accessions, as well as a domestication sweep in a diversity panel of accessions ${ }^{41}$. Additional QTLs on chromosomes 6, 7 and 11 detected in the RIL population, where the Ved allele reduced fruit size, were undetectable in our IL collection. However, the fruit shape QTL on chromosome 2 was identified in both populations. 

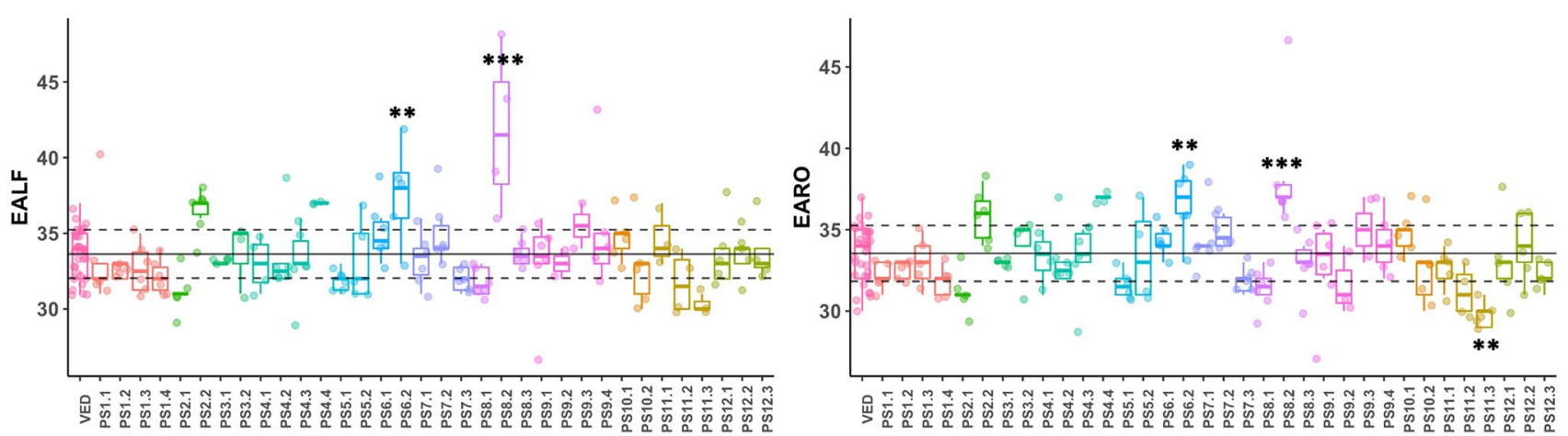

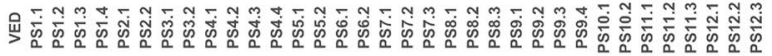
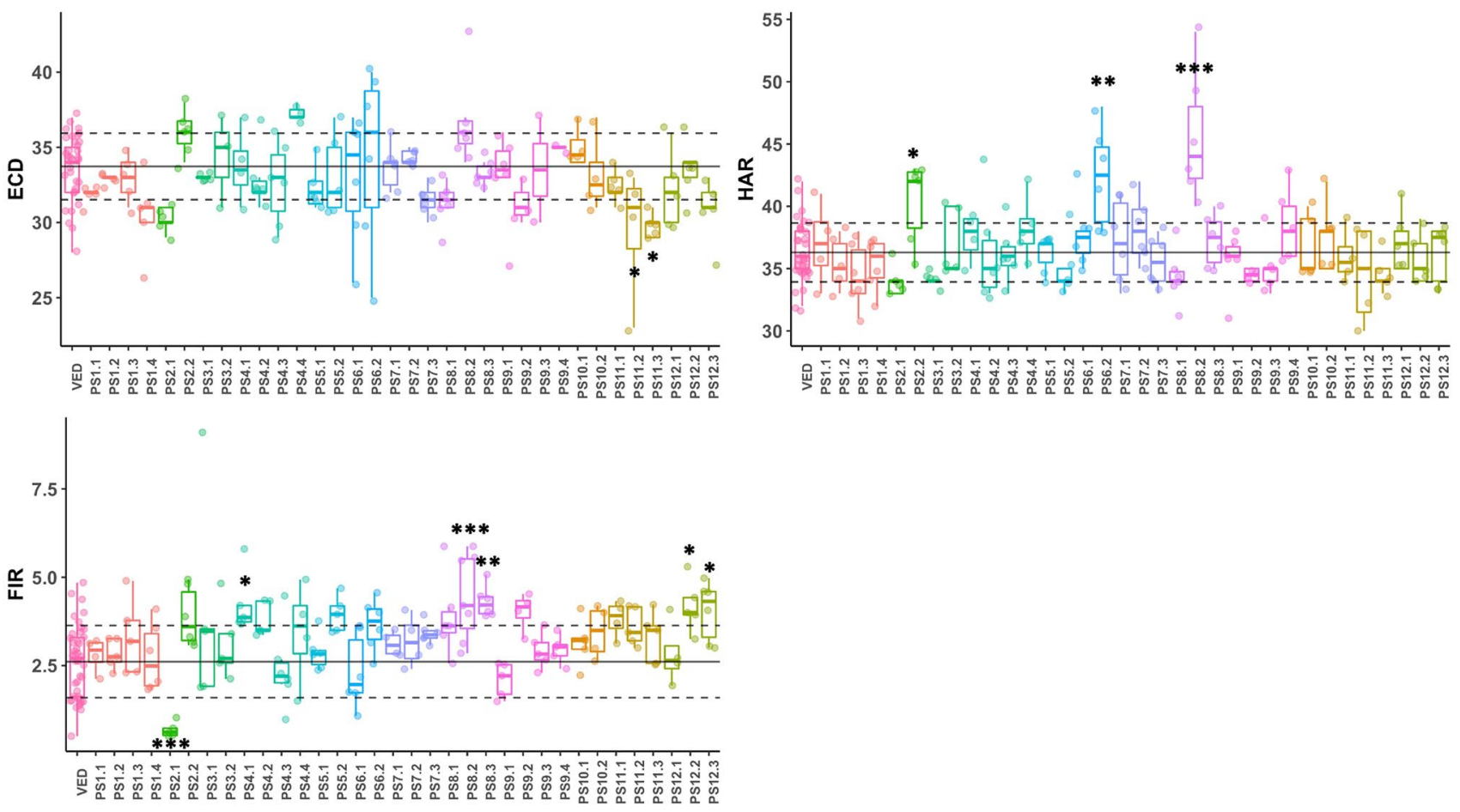

Figure 6. Boxplot representing the values of climacteric ripening traits for ILs. Each dot represents a replicate, the black solid line corresponds to the average and the grey dashed lines to the average \pm SD of the recurrent parent Ved. Significant differences are marked with asterisks: ${ }^{*}<0.05,{ }^{* *}<0.01,{ }^{* * *}<0.001$.

Fruit ripening. Melon has emerged as an alternative model to decipher the genetic control of fruit ripening, since both climacteric and non-climacteric varieties are found within the species. PS is a non-climacteric variety and Ved is highly climacteric and produces a large amount of ethylene at the onset of ripening ${ }^{54,55}$. The hypothesis of a quantitative composition of climacteric ripening, with the ethylene production and associated phenotypes varying in a continuum spectrum, rather than a qualitative presence-absence, is gaining relevance in recent studies ${ }^{9,31}$. The PS IL collection added evidence to this hypothesis, since all ILs from the collection were climacteric. Nearly all the harvested fruits were aromatic and produced abscission layer, two of the most evident and typical traits of climacteric melons. However, PS7.1 did not present consistently the change of color due to chlorophyll degradation during ripening. As mentioned before, a major gene controlling rind color in immature fruit is located on the proximal arm of chromosome 7. Both PS7.1 and PS7.2 carried the PS allele of Wi, conferring green color during fruit development. We observed that PS7.2 degraded chlorophyll and turned yellow; this phenotype is completely different from Ved, whose white color turns to cream/light orange during ripening, and from PS, whose green color is maintained during ripening. Interestingly, not all PS7.1 fruits turned to yellow but some stayed green during ripening (Supplementary Fig. S4). We defined the gene CDQP7.1 for chlorophyll degradation on chromosome 7, from the beginning of the chromosome to $2.62 \mathrm{Mb}$.

In addition to the presence-absence of climacteric symptoms, we also recorded its earliness (ECD, EARO, EALF), earliness of harvest (HAR) and flesh firmness (FIR) after harvest to quantify the intensity of climacteric ripening.

PS8.2 showed the most delayed and the less intense climacteric characteristics in the IL collection. It presented a delay of 3-8 days in the appearance of climacteric symptoms, from 33 DAP in Ved to 39 DAP in PS8.2, representing about a $20 \%$ reduction. The increase in flesh firmness was as high as $48.5 \%$. The QTL was defined from 6.89 to $25.00 \mathrm{Mb}$ on chromosome 8 (Fig. 6, Table 1 and Supplementary Fig. S3). 
PS6.2 also presented a delayed ripening of about 4 days, although the earliness of chlorophyll degradation and flesh firmness were not affected. The QTL was delimited from 7.47 to $38.30 \mathrm{Mb}$ on chromosome 6 (Fig. 6, Table 1 and Supplementary Fig. S3).

PS11.2 showed differences in ECD and PS11.3 showed differences in EARO and ECD (Fig. 6 and Table 1). Interestingly, the PS introgressions advanced the appearance of aroma and chlorophyll degradation by $\sim 3$ days, being the only QTL for which the PS allele enhances a climacteric characteristic, although the effect was subtle.

PS2.2 presented a slight yet significant delay in harvest date, representing $\sim 11 \%$ increase in comparison to Ved (Fig. 6 and Table 1). A QTL affecting earliness of ethylene production and fruit abscission was identified in the Ved $\times$ PS RIL population in a similar region of chromosome $2^{31}$.

Six lines, PS2.1, PS4.1, PS8.2, PS8.3, PS12.2 and PS12.3, differed from Ved on flesh firmness, although their climacteric behavior was otherwise unaffected. PS2.1 showed a huge decrease in flesh firmness (Fig. 6, Table 1). Cell wall degradation and the subsequent decrease of flesh firmness is only partially dependent on ethylene in melon $^{34}$ and flesh firmness in Ved and PS is similar (Supplementary Fig. S1A). So, this QTL could be the main factor controlling cell wall degradation and allowing the softening of the flesh in the non-climacteric PS. FIRQP2.1 is located at the beginning of chromosome $2(0-16.42 \mathrm{Mb})$ (Supplementary Fig. S3). The other five lines presented an increase in flesh firmness of around $~ 55-70 \%$ when compared to Ved, suggesting a complex inheritance of this trait. PS8.3 presented an increase in flesh firmness of about 65\%, similarly to PS8.2, with which shares $\sim 7 \mathrm{Mb}$. However, the overall reduction of climacteric intensity in PS8.2 was not observed in PS8.3. We cannot discern whether the FIRQP8.3 QTL causing this reduction in flesh firmness is exclusive of PS8.3 or shared between both lines, so the overlapping region was included in the interval of the QTL, namely from 25.0 to $34.77 \mathrm{Mb}$ (Supplementary Fig. S3). PS12.2 and PS12.3 also showed an increase in flesh firmness, being FIRQP12.2 located at the end of chromosome 12 (22.98-25.89 Mb). In this region, two QTLs for flesh firmness were previously described using both genome-wide association study (GWAS) and biparental mapping in melon ${ }^{56}$.

Several QTLs related to climacteric ripening have been already mapped in melon ${ }^{20,23,55}$. In the Ved $\times$ PS RIL population, several QTLs controlling or modulating ethylene production and/or climacteric traits as aroma production, fruit abscission and chlorophyll degradation have been identified ${ }^{31,32}$. In contrast with the low rate of overlapping observed for fruit morphology QTLs, all mapped QTLs affecting climacteric ripening were identified using both the Ved $\times$ PS RIL population and the PS IL collection, in similar genomic intervals ${ }^{31}$. Among them, ETHQV8.1, carried by PS8.2, was clearly and consistently the most relevant in both populations, presenting a delay in both the ethylene peak (measured only in the RIL population) and the appearance of climacteric traits (quantified in the RIL and IL collections). PS6.2 also showed a consistent delay in the climacteric related traits. A gene on chromosome 6 already cloned, MELO3C016540 (CmNAC-NOR), could be the responsible for the PS6.2 phenotype, since it delays fruit ripening and ethylene biosynthesis when mutated in a climacteric genetic background, and triggers a climacteric response in PS when introgressing the allele of the low climacteric variety PI161375.

Other melon IL collections have explored the genetic basis of fruit ripening ${ }^{20,21,23}$. In total, they mapped two more ripening QTLs on chromosomes 3, and 10. These populations shared one parent with our IL collection, and yet we were able to identify additional QTLs on chromosomes 7 (CDQP7.1 for chlorophyll degradation, Supplementary Fig. S4), 8 and 11 (several ripening traits, Table 1, Supplementary Fig. S3), supporting the complex genetic architecture of fruit ripening in melon. In addition, the partial effect of some of these QTLs, as observed in PS7.1, may be useful in breeding programs, allowing modifying only the desired trait without altering overall ripening behavior.

\section{Conclusion}

We have developed a new IL collection, using as parental lines two elite European cultivars. A RIL population derived from the same parental lines has proven its effectiveness to identify relevant fruit quality QTLs. Multiple QTLs and major genes controlling several aspects of fruit quality have been identified and the germplasm generated in this work could ease further fine mapping and positional cloning. In addition, these lines could be used to pyramid QTLs controlling complex traits such as fruit size or climacteric ripening, allowing to better understand the genetic interactions among them. Lastly, the PS IL collection may be an excellent resource for breeding programs. Generally, ILs are developed using wild or exotic material, which carry detrimental alleles by linkage drag. By using two commercial varieties of high quality and yet segregating for many relevant traits, we avoided the unfavorable alleles that could be linked to the target gene in other non-commercial materials.

\section{Materials and methods}

Plant material and breeding scheme. Seedlings were planted in fertilpots and maintained under controlled conditions in an indoor greenhouse facility (CRAG, Barcelona) during approximately three weeks. After this period, selected plants were grown in a greenhouse in Caldes de Montbui (Barcelona) during spring and summer seasons (April-October). Plants were pruned weekly and pollinations were executed manually, allowing to develop only one fruit per plant.

The IL collection was founded by a cross between two commercial varieties, "Védrantais" (Ved), a French melon from the cantalupensis group used as female, and "Piel de Sapo" T111 (PS), a Spanish melon from the inodorus group used as male. These two varieties segregate for many traits such as fruit morphology, fruit quality and ripening behavior (Table 2). Pollen from the $\mathrm{F}_{1}$ plants was used to pollinate female flowers from Ved, obtaining $\mathrm{BC}_{1}$ seed with the cytoplasm from the recurrent parent. After $\mathrm{BC}_{1}$, Ved was generally used as male and the pre-IL as female. Seedlings of the $\mathrm{BC}_{1}$ progenies were screened and a subset of plants was selected following these hierarchical criteria: (1) the complete genome of PS should be contained at least twice in the selected individuals; (2) the lines should carry the lowest possible number of introgressions; (3) the lines should contain 


\begin{tabular}{|c|c|c|}
\hline Category & Trait (units ${ }^{\mathbf{a}}$ ) & Code \\
\hline \multirow{6}{*}{ Fruit quality } & Presence of sutures & SUT \\
\hline & Rind color of immature fruit & ECOL \\
\hline & Mottled rind & MOT \\
\hline & \begin{tabular}{|l|} 
Yellowing of the rind \\
\end{tabular} & YELL \\
\hline & Soluble solids content $\left({ }^{\circ} \mathrm{Brix}\right)$ & SSC \\
\hline & Flesh color & FC \\
\hline \multirow{6}{*}{ Fruit morphology } & Fruit weight (g) & FW \\
\hline & Fruit area $\left(\mathrm{cm}^{2}\right)$ & FA \\
\hline & Fruit perimeter $(\mathrm{cm})$ & FP \\
\hline & Fruit width $(\mathrm{cm})$ & FWI \\
\hline & Fruit length $(\mathrm{cm})$ & FL \\
\hline & Fruit shape & FS \\
\hline \multirow{7}{*}{ Climacteric ripening } & Chlorophyll degradation & $\mathrm{CD}$ \\
\hline & Earliness of chlorophyll degradation (DAP) & ECD \\
\hline & Aroma production & ARO \\
\hline & Earliness of aroma production (DAP) & EARO \\
\hline & Earliness of abscission layer formation (DAP) & EALF \\
\hline & Earliness of harvesting (DAP) & HAR \\
\hline & Flesh firmness $\left(\mathrm{kg} \mathrm{cm}^{-2}\right)$ & FIR \\
\hline
\end{tabular}

Table 2. Phenotypic traits evaluated in the IL collection. ${ }^{a} D A P$ days after pollination.

the highest possible percentage of Ved genome. The chosen individuals were backcrossed again to obtain the $\mathrm{BC}_{2}$ progeny. Analogous cycles of screening and selection were performed for the subsequent progenies following the breeding scheme presented in Fig. 1. When the lines contained three or less introgressions, they were selfpollinated to identify lines carrying a unique introgression in homozygosity within the progeny. Depending on the introgression line, three or four backcrosses followed by one or two self-pollinations were needed to obtain the target introgression line in homozygosity without additional non-target introgressions in other chromosomes.

DNA extraction and genotyping. DNA extractions were performed from young leaves following the CTAB protocol ${ }^{57}$ with some modifications ${ }^{30}$.

The progenies of $\mathrm{BC}_{1}, \mathrm{BC}_{2}$ and most part of the $\mathrm{BC}_{3}$ seedlings were analyzed with an initial set of $48 \mathrm{SNPs}$ (set 1) homogeneously distributed within the 12 melon chromosomes (Supplementary Table S5a). The selected plants from $\mathrm{BC}_{2}$ and $\mathrm{BC}_{3}$ generations were subsequently genotyped with an additional set of 48 SNPs (set 2) (Supplementary Table S5b). SNPs were identified and designed from resequencing data of both parental lines ${ }^{58}$ and their positions are referred to the melon reference genome v3.6.1 (http://www.melonomics.net). SNPs producing sub-optimal genotypes were substituted by other SNPs located nearby. The progenies of $\mathrm{BC}_{3} \mathrm{~S}_{1}, \mathrm{BC}_{4}, \mathrm{BC}_{3} \mathrm{~S}_{2}$ and $\mathrm{BC}_{4} \mathrm{~S}_{1}$, screened in 2017, were genotyped with the complete set of SNPs (Supplementary Table S5c), which was comprised mostly by sets 1 and 2. Additional SNPs were used whenever necessary to genotype overlapping introgressions (Supplementary Table S5d). Plants were genotyped with SNPs using the KASPar SNP Genotyping System (KBiosciences, Herts, UK). KASPar primers were designed following LGC Genomics protocol. The genotyping was performed using the high-throughput genotyping system Biomark HD, based on the Fluidigm technology, with $48 \times 48$ (2015 and 2016) and $96 \times 96$ (2017) chips. In the last phases of screening and selection, when most part of the genome was already fixed for Ved, only SNPs contained within the known introgressions were genotyped with the same KASPar primers but using qPCR in a LightCycler 480 Real-time PCR System, according to the technical instructions provided by the supplier (Roche Diagnostics, Spain).

To calculate the size of the introgressions and the intervals delimiting the QTLs, two assumptions were done; we supposed that the haplotypes of the non-genotyped extremes of the chromosome were the same than the first or last SNP genotyped; and we used the intermediate position between two genotyped SNPs as the virtual recombination breakpoint. The approximate genetic size of the introgressions was calculated using as a reference a genetic map obtained from a Ved $\times$ PS RIL population ${ }^{30}$.

Experimental design and phenotyping of fruit quality and climacteric ripening traits. Phenotyping of most part of the IL collection, 29 ILs, was performed in greenhouse conditions in Caldes de Montbui (Barcelona) during the summer of 2018. The experiment consisted of randomized blocks including from five to seven plants of each IL and 13 plants of the recurrent parent, Ved. Three ILs that were not fully developed in 2018 were grown and phenotyped under the same conditions, along with the Ved controls, in the summer of 2019. Lastly, two more ILs were obtained in 2019 and phenotyped in 2020.

Qualitative traits related with fruit appearance were visually inspected and recorded as presence or absence (Table 2). These traits were rind color of immature fruit (ECOL), yellowing of the rind (YELL), presence of sutures (SUT) and mottled rind (MOT). Flesh color of mature fruits was recorded as orange, white or green. All 
fruits were weighed (FW), photographed and scanned at harvest. The scanned images were analyzed in Tomato Analyzer $3.0^{47}$ to extract the values of morphological traits: fruit perimeter (FP), fruit area (FA), fruit length (FL) and fruit width (FWI); fruit shape (FS) was calculated as FL/FWI. In addition to visual inspections, flesh color was also measured in scanned images in Tomato Analyzer. The CIELAB color parameters were used to estimate an index, FC, that represents the differences of each IL with respect to Ved. FC was calculated as the summatory of the subtraction of the average $a, b$ and $L$ values of the IL minus the average $a, b$ and $L$ values of Ved, respectively. To measure the soluble solid content (SSC), four 1-cm cylinders of flesh were obtained from the central part of the fruit in a proximal-distal section and the SSC value was estimated by a hand refractometer from manually extracted juice.

Ripening-related traits were evaluated as qualitative (presence or absence) or quantitative (earliness of appearance of the trait in days after pollination, DAP). The visual inspection of melon fruits attached to the plant was performed daily, from approximately 25 DAP until harvest. Chlorophyll degradation (CD) and its earliness (ECD) were recorded when degreening was obvious in visual inspections. Aroma production (ARO) and its earliness (EARO) were evaluated every other day by smelling the fruits. Earliness of abscission layer formation (EALF) was recorded as well after daily visual inspection when a scar in the pedicel was clearly formed. The harvest date was determined by the following criteria and registered (HAR): (a) abscission date when the fruit abscised; (b) after five days of the appearance of the abscission layer; (c) at 55-60 days after pollination when fruits were non-climacteric. The firmness of fruit flesh (FIR) was measured at harvest using a penetrometer (Fruit Test ${ }^{\mathrm{Tn}}$, Wagner Instruments), in at least three regions of the fruit (distal, proximal and median), and the mean value was registered.

QTL and major gene mapping and statistical analyses. A QTL was designated when the mean of one or more ILs carrying the corresponding region was significantly different to Ved mean using Dunnett's test. When two ILs overlapped in a certain region of the introgression, the overlapping interval defined the QTL if both lines were significantly different to Ved but was excluded when only one line presented the phenotype. The statistical analysis was implemented in $\mathrm{R}$ (version 3.6.3) (v) $^{5}$ using the functions aov and glht from the packages stats and multcomp, respectively. Three levels of significance $(0.05,0.01$ and 0.001$)$ were used and the p-values were specified. The genomic intervals of the QTLs and major genes were calculated using the intermediate position between two genotyped SNPs as the virtual recombination breakpoint.

Most ILs were evaluated in 2018, however three ILs were evaluated in 2019 and two more in 2020. To add these lines to the analyses, we first tested whether the recurrent parent Ved was affected by the environment. For most traits, no significant differences were observed between years. Only size-related traits (FW, FA, FP, FL and FWI) were affected. Consequently, for the morphological traits, a separated analysis was performed for each year (Fig. 5 and Supplementary Figs. S2 and S5). For SSC, FC and ripening traits (Figs. 4 and 6), the data from 2018, 2019 and 2020 were merged.

As the assumptions for applying the Dunnett test (normality of the data and equal variances) were not always accomplished (Supplementary Table S6a), we re-analyzed the data using the non-parametric Mann-Whitney test (Supplementary Table S6b). As 74\% of the QTLs detected with the Dunnett test were significant with the non-parametric test, we presented the QTLs detected with Dunnett test in Fig. 1.

Plant material. The collection and use of plant material complies with institutional, national, and international guidelines and legislation. Védrantais and Piel de Sapo T111 are melon inbred lines gently provided by Semillas Fitó SA.

\section{Data availability}

Relevant data are submitted as "Supplementary material" or are available upon request.

Received: 28 February 2021; Accepted: 18 May 2021

Published online: 31 May 2021

\section{References}

1. Harel-Beja, R. et al. A genetic map of melon highly enriched with fruit quality QTLs and EST markers, including sugar and carotenoid metabolism genes. Theor. Appl. Genet. 121, 511-533 (2010).

2. McMullen, M. D. et al. Genetic properties of the maize nested association mapping population. Science 325, 737-741 (2009).

3. Huang, B. E. et al. MAGIC populations in crops: Current status and future prospects. Theor. Appl. Genet. 128, 999-1017 (2015).

4. Eduardo, I., Arús, P. \& Monforte, A. J. Development of a genomic library of near isogenic lines (NILs) in melon (Cucumis melo L.) from the exotic accession PI161375. Theor. Appl. Genet. 112, 139-148 (2005).

5. Barrantes, W. et al. Highly efficient genomics-assisted development of a library of introgression lines of Solanum pimpinellifolium. Mol. Breed. 34, 1817-1831 (2014).

6. Díaz, A. et al. Quantitative trait loci analysis of melon (Cucumis melo L.) domestication-related traits. Theor. Appl. Genet. 130, 1837-1856 (2017)

7. Lin, H. X., Yamamoto, T., Sasaki, T. \& Yano, M. Characterization and detection of epistatic interactions of 3 QTLs, Hd1, Hd2, and Hd3, controlling heading date in rice using nearly isogenic lines. Theor. Appl. Genet. 101, 1021-1028 (2000).

8. Monforte, A. J., Friedman, E., Zamir, D. \& Tanksley, S. D. Comparison of a set of allelic QTL-NILs for chromosome 4 of tomato: Deductions about natural variation and implications for germplasm utilization. Theor. Appl. Genet. 102, 572-590 (2001).

9. Ríos, P. et al. ETHQV6.3 is involved in melon climacteric fruit ripening and is encoded by a NAC domain transcription factor. Plant J. 91, 671-683 (2017).

10. Giner, A. et al. A mutation in the melon Vacuolar Protein Sorting 41prevents systemic infection of Cucumber mosaic virus. Sci. Rep. 7, 1-12 (2017).

11. Suge, H. Effect of uzu (uz) gene on the level of endogenous gibberellins in barley. Japan. J. Genet. 47, 423-430 (1972).

12. Briggle, L. W. Near-isogenic lines of wheat with genes for resistance to Erysiphe graminis f. sp. tritici. Crop Sci. 9, 70-72 (1969). 
13. Pegg, G. F. \& Cronshaw, D. K. Ethylene production in tomato plants infected with Verticillium albo-atrum. Physiol. Plant Pathol. 8, 279-295 (1976).

14. Keurentjes, J. J. B. et al. Development of a near-isogenic line population of Arabidopsis thaliana and comparison of mapping power with a recombinant inbred line population. Genetics 175, 891-905 (2007).

15. Li, Z. K. et al. Genome-wide introgression lines and their use in genetic and molecular dissection of complex phenotypes in rice (Oryza sativa L.). Plant Mol. Biol. 59, 33-52 (2005).

16. Bernacchi, D. et al. Advanced backcross QTL analysis of tomato. II. Evaluation of near-isogenic lines carrying single-donor introgressions for desirable wild QTL-alleles derived from Lycopersicon hirsutum and L. pimpinellifolium. Theor. Appl. Genet. 97, 170-180 (1998).

17. Urrutia, M., Bonet, J., Arús, P. \& Monfort, A. A near-isogenic line (NIL) collection in diploid strawberry and its use in the genetic analysis of morphologic, phenotypic and nutritional characters. Theor. Appl. Genet. 128, 1261-1275 (2015).

18. Serra, O. et al. Marker-assisted introgression (MAI) of almond genes into the peach background: A fast method to mine and integrate novel variation from exotic sources in long intergeneration species. Tree Genet. Genomes 12, 96 (2016).

19. Foncéka, D. et al. Genetic mapping of wild introgressions into cultivated peanut: A way toward enlarging the genetic basis of a recent allotetraploid. BMC Plant Biol. 9, 1-13 (2009).

20. Perpiñá, G., Esteras, C., Gibon, Y., Monforte, A. J. \& Picó, B. A new genomic library of melon introgression lines in a cantaloupe genetic background for dissecting desirable agronomical traits. BMC Plant Biol. 16, 1-21 (2016).

21. Castro, G., Perpiñá, G., Monforte, A. J., Picó, B. \& Esteras, C. New melon introgression lines in a Piel de Sapo genetic background with desirable agronomical traits from dudaim melons. Euphytica 215, 169 (2019).

22. Perpiñá, G., Cebolla-Cornejo, J., Esteras, C., Monforte, A. J. \& Picó, B. 'MAK-10': A long shelf-life charentais breeding line developed by introgression of a genomic region from Makuwa Melon. HortScience 52, 1633-1638 (2017).

23. Vegas, J., Garcia-Mas, J. \& Monforte, A. J. Interaction between QTLs induces an advance in ethylene biosynthesis during melon fruit ripening. Theor. Appl. Genet. 126, 1531-1544 (2013).

24. Eduardo, I. et al. Estimating the genetic architecture of fruit quality traits in melon using a genomic library of near isogenic lines. J. Am. Soc. Hortic. Sci. 132, 80-89 (2007).

25. Obando-Ulloa, J. M., Ruiz, J., Monforte, A. J. \& Fernández-Trujillo, J. P. Aroma profile of a collection of near-isogenic lines of melon (Cucumis melo L.). Food Chem. 118, 815-822 (2010).

26. Obando-Ulloa, J. M., Eduardo, I., Monforte, A. J. \& Fernández-Trujillo, J. P. Identification of QTLs related to sugar and organic acid composition in melon using near-isogenic lines. Sci. Hortic. 121, 425-433 (2009).

27. Garcia-Mas, J. et al. The genome of melon (Cucumis melo L.). Proc. Natl. Acad. Sci. USA 109, 11872-11877 (2012).

28. Ezura, H. \& Owino, W. O. Melon, an alternative model plant for elucidating fruit ripening. Plant Sci. 175, 121-129 (2008).

29. Martin, A. et al. A transposon-induced epigenetic change leads to sex determination in melon. Nature 461, 1135-1138 (2009).

30. Pereira, L. et al. QTL mapping of melon fruit quality traits using a high-density GBS-based genetic map. BMC Plant Biol. https:// doi.org/10.1186/s12870-018-1537-5 (2018).

31. Pereira, L. et al. Genetic dissection of climacteric fruit ripening in a melon population segregating for ripening behavior. Hortic. Res. https://doi.org/10.1038/s41438-020-00411-z (2020).

32. Mayobre, C. et al. Genetic dissection of aroma biosynthesis in melon and its relationship with climacteric ripening. Food Chem. 353, $129484(2021)$.

33. Lelièvre, J.-M., Latchè, A., Jones, B., Bouzayen, M. \& Pech, J.-C. Ethylene and fruit ripening. Physiol. Plant. 101, 727-739 (1997).

34. Pech, J. C., Bouzayen, M. \& Latché, A. Climacteric fruit ripening: Ethylene-dependent and independent regulation of ripening pathways in melon fruit. Plant Sci. 175, 114-120 (2008).

35. Urrutia, M., Rambla, J. L., Alexiou, K. G., Granell, A. \& Monfort, A. Genetic analysis of the wild strawberry (Fragaria vesca) volatile composition. Plant Physiol. Biochem. 121, 99-117 (2017).

36. Barrantes, W. et al. Exploring new alleles involved in tomato fruit quality in an introgression line library of solanum pimpinellifolium. Front. Plant Sci. 7, 1-12 (2016).

37. Argyris, J. M. et al. QTL analyses in multiple populations employed for the fine mapping and identification of candidate genes at a locus affecting sugar accumulation in melon (Cucumis melo L.). Front. Plant Sci. 8, 1-20 (2017).

38. Périn, C. et al. Genetic control and linkages of some fruit characters in melon. Cucurbit Genet. Coop. Rep. 22, 16-18 (1999).

39. Kubicki, B. Inheritance of some characters in muskmelons (Cucumis melo). Genet Pol 3, 265-274 (1962).

40. Feder, A. et al. A Kelch domain-containing F-box coding gene negatively regulates flavonoid accumulation in muskmelon. Plant Physiol. 169, 1714-1726 (2015).

41. Zhao, G. et al. A comprehensive genome variation map of melon identifies multiple domestication events and loci influencing agronomic traits. Nat. Genet. 51, 1607-1615 (2019).

42. Esteras, C. et al. High-throughput SNP genotyping in Cucurbita pepo for map construction and quantitative trait loci mapping. BMC Genom. 13, 80 (2012).

43. Tzuri, G. et al. A 'golden' SNP in CmOr governs the fruit flesh color of melon (Cucumis melo). Plant J. 82, 267-279 (2015).

44. Galpaz, N. et al. Deciphering genetic factors that determine melon fruit-quality traits using RNA-Seq-based high-resolution QTL and eQTL mapping. Plant J. 94, 169-191 (2018).

45. Cuevas, H. E., Staub, J. E., Simon, P. W. \& Zalapa, J. E. A consensus linkage map identifies genomic regions controlling fruit maturity and beta-carotene-associated flesh color in melon (Cucumis melo L.). Theor. Appl. Genet. 119, 741-756 (2009).

46. Monforte, A. J. et al. Identification of quantitative trait loci involved in fruit quality traits in melon (Cucumis melo L.). Theor. Appl. Genet. 108, 750-758 (2004).

47. Rodríguez, G. R. et al. Tomato analyzer: A useful software application to collect accurate and detailed morphological and colorimetric data from two-dimensional objects. JoVE. https://doi.org/10.3791/1856 (2010).

48. Mu, Q. et al. Fruit weight is controlled by Cell Size Regulator encoding a novel protein that is expressed in maturing tomato fruits. PLoS Genet. 13, 1-26 (2017).

49. Frary, A. et al. fw2.2: A quantitative trait locus key to the evolution of tomato fruit size. Science 289, 85-88 (2000).

50. Chakrabarti, M. et al. A cytochrome P450 regulates a domestication trait in cultivated tomato. Proc. Natl. Acad. Sci. USA. 110, $17125-17130$ (2013).

51. Monforte, A. J., Diaz, A., Caño-Delgado, A. \& Van Der Knaap, E. The genetic basis of fruit morphology in horticultural crops: Lessons from tomato and melon. J. Exp. Bot. 65, 4625-4637 (2014).

52. Boualem, A. et al. A conserved mutation in an ethylene biosynthesis enzyme leads to andromonoecy in melons. Science 321, $836-838$ (2008).

53. Amanullah, S. et al. Genetic linkage mapping and QTLs identification for morphology and fruit quality related traits of melon by SNP based CAPS markers. Sci. Hortic. 278, 109849 (2021).

54. Saladié, M. et al. Comparative transcriptional profiling analysis of developing melon (Cucumis melo L.) fruit from climacteric and non-climacteric varieties. BMC Genom. 16, 1-20 (2015).

55. Périn, C. et al. Molecular and genetic characterization of a non-climacteric phenotype in melon reveals two loci conferring altered ethylene response in fruit. Plant Physiol. 129, 300-309 (2002).

56. Nimmakayala, P. et al. Genome-wide differentiation of various melon horticultural groups for use in GWAS for fruit firmness and construction of a high resolution genetic map. Front. Plant Sci. 7, 1437. https://doi.org/10.3389/fpls.2016.01437 (2016). 
57. Doyle, J. DNA protocols for plants. In Molecular Techniques in Taxonomy (eds Hewitt, G. M. et al.) 283-293 (Springer Berlin Heidelberg, 1991). https://doi.org/10.1007/978-3-642-83962-7_18.

58. Sanseverino, W. et al. Transposon insertions, structural variations, and SNPs contribute to the evolution of the melon genome. Mol. Biol. Evol. 32, 2760-2774 (2015).

59. The R Core Team. R: A Language and Environment for Statistical Computing (R Foundation for Statistical Computing, 2019).

\section{Acknowledgements}

This work was supported by the Spanish Ministry of Economy and Competitiveness Grants AGL2015-64625C2-1-R and RTI2018-097665-B-C2, Severo Ochoa Programme for Centres of Excellence in R\&D 2016-2010 (SEV-2015-0533) and the CERCA Programme/Generalitat de Catalunya to J.G.-M. L.P., L.V. and M.S.D. were supported by a FPI grant from the Spanish Ministry of Economy and Competitiveness, and CM was supported by 2019 FI_B 00124 project from the Secretaria d'Universitats i Recerca del Departament d'Empresa i Coneixement de la Generalitat de Catalunya and the co-funding of the European Social Fund (ESF- "ESF is investing in your future") from the European Union.

\section{Author contributions}

M.P. and J.G.-M. conceived and designed the research. L.P., M.S.D., J.A., C.M. and L.V. performed the experiments and data analyses. L.P. wrote the original draft. M.S.D., M.P. and J.G.-M. reviewed and edited the manuscript. A.M.M.-H. and J.G.-M. contributed to funding acquisition. All authors read and approved the manuscript.

\section{Competing interests}

The authors declare no competing interests.

\section{Additional information}

Supplementary Information The online version contains supplementary material available at https://doi.org/ 10.1038/s41598-021-90783-6.

Correspondence and requests for materials should be addressed to M.P. or J.G.-M.

Reprints and permissions information is available at www.nature.com/reprints.

Publisher's note Springer Nature remains neutral with regard to jurisdictional claims in published maps and institutional affiliations.

Open Access This article is licensed under a Creative Commons Attribution 4.0 International License, which permits use, sharing, adaptation, distribution and reproduction in any medium or format, as long as you give appropriate credit to the original author(s) and the source, provide a link to the Creative Commons licence, and indicate if changes were made. The images or other third party material in this article are included in the article's Creative Commons licence, unless indicated otherwise in a credit line to the material. If material is not included in the article's Creative Commons licence and your intended use is not permitted by statutory regulation or exceeds the permitted use, you will need to obtain permission directly from the copyright holder. To view a copy of this licence, visit http://creativecommons.org/licenses/by/4.0/.

(C) The Author(s) 2021 